RESIDENT

\& FELLOW

SECTION

Section Editor

Mitchell S.V. Elkind,

MD, MS

Teresa Buracchio, MD

Janet C. Rucker, MD

Address correspondence and reprint requests to Dr. Janet C. Rucker, Department of Neurological Sciences, Rush University Medical Center, 1725 West Harrison Street, Suite 1106, Chicago, IL 60612 janet_rucker@rush.edu

\section{Pearls and oy-sters of localization in ophthalmoparesis}

(]

\section{ABSTRACT}

Ocular misalignment and ophthalmoparesis result in the symptom of binocular diplopia. In the evaluation of diplopia, localization of the ocular motility disorder is the main objective. This requires a systematic approach and knowledge of the ocular motor pathways and actions of the extraocular muscles. This article reviews the components of the ocular motor pathway and presents helpful tools for localization and common sources of error in the assessment of ophthalmoparesis. Neurology ${ }^{\circledR}$ 2007;69:E35-E40

Ophthalmoparesis and diplopia. Normal eye movements share the goal of placing an object of visual interest on each fovea simultaneously to allow visualization of a single, stable object. Clear and stable vision is sustained by mechanisms that hold the object on the fovea, such as fixation and the vestibuloocular reflex. Absent or inadequate ocular motility (ophthalmoplegia and ophthalmoparesis) often results in ocular misalignment, causing the visual symptom of binocular diplopia. Binocular diplopia occurs when an object of visual interest falls on the fovea in one eye and on an extrafoveal location in the other eye. Binocular diplopia suggests dysfunction of extraocular muscles, the neuromuscular junction, cranial nerves, cranial nerve nuclei, or internuclear and supranuclear connections. Correct localization of the underlying lesion is the first step to accurate diagnosis and requires a systematic approach and knowledge of the ocular motor pathways and actions of the extraocular muscles.

History and examination of diplopia. When obtaining the history and examining the patient, it is important to determine if the diplopia is binocular or monocular. Binocular diplopia resolves with covering either eye and is the result of ocular misalignment. Proper evaluation of binocular diplopia should determine if it is horizontal, vertical, or oblique; worse in a particular direction of gaze; and worse at distance or near. Eye movement examination should include assessment of ocular motility in the nine diagnostic positions of gaze, ocular alignment (measured with the corneal light reflex test, cover test, or Maddox $\operatorname{rod}^{1}$ ), and comitance of any ocular misalignment. In a comitant lesion, the amount of ocular deviation is the same regardless of gaze direction, while in an incomitant lesion, the amount of deviation varies with changes in gaze direction.

Pearls

- Binocular diplopia resolves with monocular covering of either eye, while monocular diplopia resolves with covering the affected eye.

- Visual blurring that resolves completely with monocular covering of either eye has the same localizing value as binocular diplopia.

- Monocular diplopia is non-neurologic in origin and is not caused by ocular misalignment. It is usually due to ocular pathology such as refractive error or intraocular causes such as cataracts. ${ }^{2}$

- Worsening diplopia in a particular gaze direction suggests that motility in that direction is impaired.

- Esodeviation is a relative medial deviation of the eyes. Exodeviation is a relative lateral deviation of the eyes. Hyperdeviation is a relative elevation of one eye.

- A tropia is a visually obvious deviation. A phoria is deviation visible only upon interruption of binocular fusion by covering either eye.

From the Departments of Neurology (T.B., J.C.R.) and Ophthalmology (J.C.R.), Rush University Medical Center, Chicago, IL. Disclosure: The authors report no conflicts of interest. 
- Horizontal diplopia is caused by impaired abduction or adduction of one or both eyes and vertical diplopia by impaired elevation or depression.

- Diplopia worse at distance accompanies impaired abduction or divergence.

- Diplopia worse at near accompanies impaired adduction or convergence.

- Most neurologic ocular misalignments are incomitant.

\section{Oy-sters and pitfalls}

- Patients with severe monocular visual loss (generally worse than 20/200) will not experience binocular diplopia with ocular misalignment.

- Displacement or "dragging" of the fovea is a rare cause of binocular diplopia in the absence of an ocular misalignment. It is due to macular disease such as an epiretinal membrane that may wrinkle or scar with subsequent distortion of the macula. This may displace the fovea resulting in an extrafoveal image in one eye and binocular diplopia. ${ }^{3,4}$ Monocular blurred vision or a history of macular disease may be clues.

- Cerebral polyopia is a form of cortical visual perseveration from parieto-occipital pathology. It refers to two or more visual images persistent with monocular covering and is often accompanied by a homonymous hemianopia.

Extraocular muscles. Inflammation or infiltration of individual eye muscles may cause binocular diplopia through a restrictive process. Thyroid eye disease, idiopathic orbital inflammation (orbital pseudotumor), and malignant infiltration are the most common orbital diseases of this type. See table 1 for a list of signs of orbital disease.

Pearls

- Inflammation of an extraocular muscle typically results in impaired eye movement in the direction away from the muscle because it restricts muscle movement. This is in contrast to cranial nerve dysfunction in which weakness of an extraocular muscle from decreased innervation results in impaired motility in the direction of action of the weak muscle.

- Thyroid eye disease may occur in the absence of symptoms or a previous history of thyroid disease and even with normal thyroid studies.

- Lid retraction is common in extraocular muscle disease.
Table 1 Signs of extraocular muscle involvement in ophthalmoparesis

Periorbital edema

Conjunctival injection and chemosis

Proptosis

Eyelid retraction

Lagophthalmos (inability to close the eye completely)

Lid lag (higher than normal lid position in downgaze)

von Graefe sign (dynamic slowing of lid descent during eye movement from upgaze into downgaze)

Optic neuropathy (from optic nerve compression)

- Chronic progressive external ophthalmoplegia (CPEO) from extraocular muscle myopathy may cause painless, progressive ophthalmoparesis and unilateral or bilateral ptosis.

- Mitochondrial myopathy is the most common etiology of CPEO. It may be isolated or part of a syndrome such as Kearns-Sayre.

\section{$\mathrm{O} y$-sters and pitfalls}

- External signs of orbital disease may be minimal in some patients.

- Diplopia may be absent if a disease process is bilateral and symmetric as in CPEO.

Neuromuscular junction. Myasthenia gravis (MG) is the most common disease of the neuromuscular junction. It may cause nearly any abnormal eye movement. Table 2 lists examination findings suggestive of MG. A positive edrophonium chloride test provides diagnostic support for MG. Edrophonium chloride is a reversible acetylcholinesterase inhibitor that decreases breakdown of acetylcholine in the synaptic cleft, thereby improving neuromuscular transmission. Up to 10 $\mathrm{mg}$ of edrophonium chloride is administered in small increments while the patient's cardiac status is closely monitored. A positive test demonstrates objective improvement in weakness or ptosis on examination within several minutes after injection of the edrophonium. Alternatives to the edrophonium test are the ice pack test and the rest test. In the ice pack test, an ice pack is placed over a closed ptotic eye for 2 minutes, followed by observation for improvement. The premise is that neuromuscular transmission is improved by cold temperatures. In the rest test, observation for improvement follows a period of 2 minutes during which the eyes are closed and at rest. Standard treatments for MG may be used, but optimal treatment of ocular MG is unclear.

\section{Pearls}

- The pupil is not involved in MG. 
Table 2 Examination findings in ocular MG

Cogan's lid twitch

Following a few seconds of sustained downgaze, saccadic return of the eyes to central position is accompanied by initial elevation upper eyelid, twitching, and then the return of ptosis.

Peek sign

Prolonged eye closure increases orbicularis oculi weakness and causes lid separation and globe exposure despite initial complete eye closure.

Enhanced ptosis

Lid retraction

Ice pack test

Rest test non-ptotic eyelid upon manual elevation of the more ptotic lid.

Maximal innervation to keep a retraction.

An ice pack is placed over a followed by observation for improvement.

Improvement in
Increased ptosis in a less or ptotic lid open results in excess contralateral innervation and lid closed ptotic eye for 2 minutes, ophthalmoplegia or ptosis following a period of rest.

- MG may mimic any pupil-sparing ocular motility disorder.

- Evaluation of eye movements in suspected MG should include maintained upgaze for at least 2 minutes to adequately assess for the appearance or worsening of ptosis or downdrift of the elevated eye.

- Hering's law of equal neural innervation to both eyelids is the basis for the finding of lid retraction and enhanced ptosis. Maximal innervation to keep a ptotic lid open results in excess contralateral innervation and may minimize contralateral ptosis or create an appearance of lid retraction.

- In the diagnostic sign of enhanced ptosis, manual elevation of the severely ptotic lid decreases the innervation to both lids. As a result, contralateral ptosis becomes more prominent.

\section{Oy-sters and pitfalls}

- Clostridium botulinum neurotoxin blockade of neuromuscular transmission may mimic the oculomotor features of MG, but unlike MG, it may involve the pupil.

- Miller Fisher syndrome causes demyelination of ocular motor cranial nerve roots and may also cause diffuse ophthalmoplegia, mimicking myasthenia. Diagnostic clues include the presence of areflexia and ataxia, although isolated ophthalmoplegia is reported. . $^{5,6}$

- The finding of lid retraction should prompt consideration of coexisting thyroid eye dis- ease, especially if proptosis is present. Thyroid disease occurs with a higher incidence in MG, particularly in the presence of acetylcholine receptor antibodies and in patients with ocular MG. ${ }^{7,8}$

- A positive edrophonium chloride test is useful only when a defined deficit such as significant ptosis or a fixed oculomotor defect may be monitored for test response.

Cranial nerves. Cranial nerve palsies result in ophthalmoparesis in the direction of action of the weak muscle. Lesions may occur anywhere along the course of the cranial nerve and may affect multiple cranial nerves. Cavernous sinus lesions may affect cranial nerves three, four, the first and second divisions of five, six, and sympathetic fibers. Orbital apex lesions may affect cranial nerves three, four, the first division of five, six, and the optic nerve with associated vision loss. The association of vision loss with ophthalmoparesis is critically important in localizing a lesion to the orbital apex. Inflammation (idiopathic or from systemic disorders), malignant neoplastic infiltration, meningioma, and internal carotid artery aneurysms are common lesions in the cavernous sinus. Lesions at the orbital apex include idiopathic (orbital pseudotumor) and systemic inflammation, infection (mucormycosis and aspergillus), and malignancy.

Third nerve (oculomotor nerve). The third cranial nerve originates in the dorsal midbrain, exits the brainstem ventrally, traverses the subarachnoid space to reach the cavernous sinus, and enters the orbit via the superior orbital fissure. Just prior to entry, it divides into a superior branch that innervates the levator palpebrae superioris and the superior rectus and an inferior branch that innervates the inferior and medial recti, the inferior oblique, and the iris sphincter and ciliary muscles.

\section{Pearls}

- In a complete pupil-involved third nerve palsy, the eye is deviated down and out, with impaired elevation, depression, and adduction. The pupil is dilated and there is ptosis. Posterior communicating artery (PCOM) aneurysm is the etiology until proven otherwise. Neuroimaging is indicated in these patients with CT angiography (CTA), MRI or angiography (MRI/MRA), or cerebral angiography if other neuroimaging modalities are negative and suspicion is high. 
- In a complete pupil-sparing third nerve palsy, all third nerve muscles are impaired and there is ptosis. This is very unlikely to be caused by a PCOM aneurysm. Microvascular ischemia is a common cause. Neuroimaging is not absolutely indicated in these patients, although measurement of glucose and sedimentation rate and close observation are indicated. Neuroimaging to look for an underlying lesion is absolutely indicated if a presumed microvascular third nerve palsy fails to spontaneously resolve in 8 to 12 weeks.

- The presence or absence of pupillary dysfunction is an important feature in the identification of an incomplete third nerve palsy. Pupil involvement usually indicates a compressive lesion, while sparing of the pupil is more likely ischemia. However, neuroimaging is generally recommended in these patients given the morbidity and mortality risk of missing an aneurysm, particularly if the patient is younger than 50, given the lower risk of an ischemic lesion in that age group.

- Aberrant regeneration after a third nerve injury causes ipsilateral elevation of the eyelid or constriction of the pupil during adduction or depression of the eye.

- Aberrant regeneration following an acute third nerve palsy suggests a compressive PCOM aneurysm or traumatic etiology.

- Spontaneous aberrant regeneration without a pre-existing acute third nerve palsy suggests a cavernous sinus meningioma or an internal carotid artery aneurysm.

\section{$\mathrm{O} y$-sters and pitfalls}

- Primary aberrant regeneration may occur rarely with an unruptured PCOM aneurysm. ${ }^{9}$

- When a third nerve palsy follows minor head trauma, neuroimaging is indicated to evaluate for an underlying PCOM aneurysm.

Fourth nerve (trochlear nerve). The trochlear nerve originates in the dorsal midbrain just inferior to the inferior colliculus, exits the brainstem dorsally, decussates within the anterior medullary velum, and wraps around the midbrain to the ventral surface within the subarachnoid space. It then enters the cavernous sinus, where it is located within the dural sinus wall. The nerve then enters the superior orbital fissure and innervates the superior oblique muscle contralateral to its nucleus of origin.
Pearls

- The trochlear nerve innervates the superior oblique muscle and its primary action is intorsion of the eye. Its secondary action is depression of the adducted eye.

- Trochlear nerve dysfunction results in hypertropia of the affected eye and vertical diplopia.

- The hypertropia and diplopia are more prominent with downgaze and gaze in the direction contralateral to the affected side.

- Diplopia is minimized when a contralateral head tilt in the direction away from the paretic eye places the affected eye in an extorted position.

- Evaluation of superior oblique function in the setting of an oculomotor palsy with impaired adduction is challenging. It is best assessed with the affected eye in abducted position where intact intorsion during downgaze suggests preserved superior oblique function.

- Common causes of trochlear nerve lesions include congenital, trauma, infection, inflammation, ischemia, or neoplasm. Within the brainstem, stroke or neoplasm are common causes but seldom present as isolated fourth nerve palsies.

- The dorsal decussation near the tentorium cerebelli makes the trochlear nerves particularly prone to traumatic injury. ${ }^{10}$

- A lesion within the subarachnoid portion of the nerve may result from meningitis or a trochlear nerve schwannoma. ${ }^{11}$

\section{Oy-sters and pitfalls}

- Old photographs of the patient that show a head tilt may suggest long-standing misalignment such as that seen with congenital fourth nerve dysfunction.

- The trochlear fascicle within the brainstem is an uncommon site of pathology, given its short course and dorsal exit. When it does occur, paresis of the superior oblique is contralateral to the lesion.

- Another common cause of vertical diplopia is skew deviation, due to asymmetric vestibular input into ocular motor nuclei.

Sixth nerve palsy (abducens nerve). The abducens nerve originates in the caudal pons, exits the brainstem ventrally, and travels in the subarachnoid space, where it ascends near the clivus. It pierces the dura and passes under the petroclinoid (Gruber's) ligament in Dorello's canal, then passes through the body of the cavernous sinus 
(unlike the oculomotor and trochlear nerves housed in the dural sinus wall), ultimately entering the superior orbital fissure to innervate the lateral rectus.

Pearls

- Abducens dysfunction results in ipsilateral abduction paresis and esotropia.

- Meningitis may cause an abducens palsy, as may an inflammatory or neoplastic process of the clivus.

- The abducens nerves are prone to dysfunction from increased or decreased intracranial pressure. Tethering of the nerve in Dorello's canal renders it susceptible to distortion and stretch injury from such alterations in intracranial pressure. ${ }^{12}$

- Microvascular ischemia may cause an acuteonset, isolated sixth nerve palsy that should spontaneously resolve over 8 to 12 weeks. This process is often painful and may be severe. Pain, however, may be absent and its presence is nonspecific.

\section{$\mathrm{Oy}$-sters and pitfalls}

- Common sixth nerve mimics include MG and restrictive medial rectus involvement in thyroid eye disease.

- Additional diagnostic evaluation for other etiologies is required when a presumed microvascular sixth nerve palsy fails to resolve spontaneously.

Ocular motor nerve nuclei. Ocular motor nerve nuclear lesions differ in appearance from their corresponding cranial neuropathies.

\section{Pearls}

- The oculomotor nucleus provides bilateral innervation to the superior recti and a single midline levator palpebrae subnucleus innervates both levator muscles. Therefore, an oculomotor nuclear lesion classically causes ipsilateral ophthalmoparesis and pupil dilatation with bilateral impairment of ocular elevation and bilateral ptosis.

- A trochlear nuclear lesion causes a contralateral superior oblique palsy and an ipsilateral Horner syndrome because of the proximity of the preganglionic sympathetic fibers to the dorsally placed fourth nucleus.

- An abducens nuclear lesion causes ipsilateral horizontal gaze palsy because the abducens nucleus contains both abducens motoneurons and interneurons that are destined to ascend in the contralateral medial longitudi- nal fasciculus (MLF) to the contralateral oculomotor medial rectus subnucleus.

- Common etiologies of ocular motor nuclear lesions include demyelination, infarction, and Wernicke encephalopathy.

\section{$\mathrm{O} y$-sters and pitfalls}

- Rare cases of isolated horizontal gaze palsy exist, ${ }^{13}$ but the gaze palsy is more typically accompanied by an ipsilateral facial nerve palsy because the seventh cranial nerve fascicle wraps around the sixth nerve nucleus.

- Due to the functional division of the subnuclei, isolated bilateral ptosis or isolated weakness of a single muscle such as the superior rectus are also possible with an oculomotor nuclear lesion. ${ }^{14,15}$

Internuclear ophthalmoplegia. Internuclear ophthalmoplegia (INO) is caused by a lesion of the MLF, which carries signals from the abducens nucleus to the contralateral medial rectus oculomotor subnucleus. The abducens nerve and MLF coordinate conjugate horizontal eye movements with co-contraction of the ipsilateral lateral rectus and contralateral medial rectus muscles.

Pearls

- Classic signs of a unilateral INO include impaired adduction of the ipsilesional eye and abducting nystagmus of the contralateral eye.

- Multiple sclerosis and microvascular brainstem ischemia are the most common causes of INO. The two causes may be distinguished by age at presentation, with younger patients likely to have demyelination and older patients ischemia. ${ }^{16,17}$

\section{Oy-sters and pitfalls}

- Despite ipsilateral adduction weakness with direct motility testing, adduction is often intact with convergence since vergence signals to the medial rectus motoneurons are distinct from the MLF. ${ }^{18}$

- Smooth pursuit may be normal and INO diagnosed only by the presence of decreased velocity of the adducting eye (adduction lag) during saccadic testing. ${ }^{19}$

- A "pseudo-INO" may occur in MG.

Supranuclear. Supranuclear eye movement abnormalities result from dysfunctional cerebral, cerebellar, and brainstem afferent connections to the ocular motor nuclei. Burst neurons in the brainstem provide sudden, intense neural discharges required to initiate high velocity saccades. Burst neurons for horizontal saccades are located in the 
pontine paramedian reticular formation (PPRF) and, for vertical saccades, in the midbrain rostral interstitial medial longitudinal fasciculus (riMLF). A PPRF lesion causes slow or absent horizontal saccades, while a riMLF lesion causes slow or absent vertical saccades. Burst inhibition, required to prevent unwanted saccades from degrading vision, is provided by pontine omnipause neurons.

\section{Pearls}

- A clinical hallmark of supranuclear eye movement disorders is disproportionate involvement of saccades. Vestibular eye movements are typically spared.

- In contrast, nuclear and infranuclear lesions impair saccades, smooth pursuit, and vestibular eye movements equally.

- Progressive supranuclear palsy is a common cause of supranuclear gaze palsy, initially affecting vertical gaze to a greater extent than horizontal.

- The dorsal midbrain (Parinaud) syndrome is comprised of a supranuclear upgaze palsy, convergence-retraction nystagmus, Collier sign (lid retraction), and pupillary light-near dissociation. The most common etiologies are pineal gland lesions and hydrocephalus.

- Gaze deviation is a common supranuclear process. The frontal eye fields (FEF) project to the contralateral PPRF. A destructive lesion (e.g., stroke) of the FEF results in ipsiversive bilateral gaze deviation: the patient "looks at the lesion." With an irritative FEF lesion (e.g., hemorrhage or seizure), there is contraversive deviation: the patient "looks away from the lesion."

\section{Oy-sters and pitfalls}

- A supranuclear upgaze palsy with forced downward deviation of the eyes ("peering at the tip of the nose") may result from a thalamic lesion; however, most of these purported thalamic lesions extend into the midbrain. ${ }^{20}$

- Thalamic lesions may also cause supranuclear thalamic esotropia, likely secondary to excessive convergence tone.

- "Wrong way eyes" with the patient "looking away" from a destructive lesion may occur from thalamic pathology. ${ }^{21}$

\section{REFERENCES}

1. Rucker JC, Tomsak RL. Binocular diplopia. A practical approach. Neurologist 2005;11:98-110.

2. Coffeen P, Guyton DL. Monocular diplopia accompanying ordinary refractive errors. Am J Ophthalmol 1988;105:451-459.
3. Barton JJ. "Retinal diplopia" associated with macular wrinkling. Neurology 2004;63:925-927.

4. De Pool ME, Campbell JP, Broome SO, Guyton DL. The dragged-fovea diplopia syndrome: clinical characteristics, diagnosis, and treatment. Ophthalmology 2005;112:1455-1462.

5. Yuki N. Acute paresis of extraocular muscles associated with IgG anti-GQ1b antibody. Ann Neurol 1996; 39:668-672.

6. Chan Y, Wilder-Smith E, Chee M. Acute ophthalmoplegia with pupillary areflexia associated with antiGQ1b antibody. J Clin Neurosci 2004;11:658-660.

7. Toth C, McDonald D, Oger J, Brownell K. Acetylcholine receptor antibodies in myasthenia gravis are associated with greater risk of diabetes and thyroid disease. Acta Neurol Scand 2006;114:124-132.

8. Marino M, Barbesino G, Pinchera A, et al. Increased frequency of euthyroid ophthalmopathy in patients with Graves' disease associated with myasthenia gravis. Thyroid 2000;10:799-802.

9. Carrasco JR, Savino PJ, Bilyk JR. Primary aberrant oculomotor nerve regeneration from a posterior communicating artery aneurysm. Arch Ophthalmol 2002;120: 663-665.

10. Keane JR. Bilateral involvement of a single cranial nerve: analysis of 578 cases. Neurology 2005;65:950-952.

11. Feinberg AS, Newman NJ. Schwannoma in patients with isolated unilateral trochlear nerve palsy. Am J Ophthalmol 1999;127:183-188.

12. Hanson RA, Ghosh S, Gonzalez-Gomez I, Levy ML, Gilles FH. Abducens length and vulnerability? Neurology 2004;62:33-36.

13. Miller NR, Biousse V, Hwang T, Patel S, Newman NJ, Zee DS. Isolated acquired unilateral horizontal gaze paresis from a putative lesion of the abducens nucleus. J Neuroophthalmol 2002;22:204-207.

14. Rabadi MH, Beltmann MA. Midbrain infarction presenting isolated medial rectus nuclear palsy. Am J Med 2005;118:836-837.

15. Kwon JH, Kwon SU, Ahn HS, Sung KB, Kim JS. Isolated superior rectus palsy due to contralateral midbrain infarction. Arch Neurol 2003;60:1633-1635.

16. Kim JS. Internuclear ophthalmoplegia as an isolated or predominant symptom of brainstem infarction. Neurology 2004;62:1491-1496.

17. Bolanos I, Lozano D, Cantu C. Internuclear ophthalmoplegia: causes and long-term follow-up in 65 patients. Acta Neurol Scand 2004;110:161-165.

18. Gamlin PD, Gnadt JW, Mays LE. Lidocaine-induced unilateral internuclear ophthalmoplegia: effects on convergence and conjugate eye movements. J Neurophysiol 1989;62:82-95.

19. Frohman TC, Frohman EM, O'Suilleabhain P, et al. Accuracy of clinical detection of INO in MS: corroboration with quantitative infrared oculography. Neurology 2003;61:848-850.

20. Choi KD, Jung DS, Kim JS. Specificity of "peering at the tip of the nose" for a diagnosis of thalamic hemorrhage. Arch Neurol 2004;61:417-422.

21. Messe SR, Cucchiara BL. Wrong-way eyes with thalamic hemorrhage. Neurology 2003;60:1524. 


\title{
Neurology
}

\author{
Pearls and oy-sters of localization in ophthalmoparesis \\ Teresa Buracchio and Janet C. Rucker \\ Neurology 2007;69;E35-E40 \\ DOI 10.1212/01.wnl.0000291013.26249.74
}

\section{This information is current as of December 10, 2007}

\section{Updated Information \& Services}

References

Citations

Subspecialty Collections

Permissions \& Licensing

Reprints including high resolution figures, can be found at: http://n.neurology.org/content/69/24/E35.full

This article cites 21 articles, 6 of which you can access for free at: http://n.neurology.org/content/69/24/E35.full\#ref-list-1

This article has been cited by 2 HighWire-hosted articles: http://n.neurology.org/content/69/24/E35.full\#\#otherarticles

This article, along with others on similar topics, appears in the following collection(s):

Clinical neurology examination

http://n.neurology.org/cgi/collection/clinical_neurology_examination

Clinical neurology history

http://n.neurology.org/cgi/collection/clinical_neurology_history

Diplopia (double vision)

http://n.neurology.org/cgi/collection/diplopia_double_vision

Ocular motility

http://n.neurology.org/cgi/collection/ocular_motility

Information about reproducing this article in parts (figures,tables) or in its entirety can be found online at:

http://www.neurology.org/about/about_the_journal\#permissions

Information about ordering reprints can be found online:

http://n.neurology.org/subscribers/advertise

Neurology ${ }^{\circledR}$ is the official journal of the American Academy of Neurology. Published continuously since 1951, it is now a weekly with 48 issues per year. Copyright . All rights reserved. Print ISSN: 0028-3878. Online ISSN: 1526-632X.

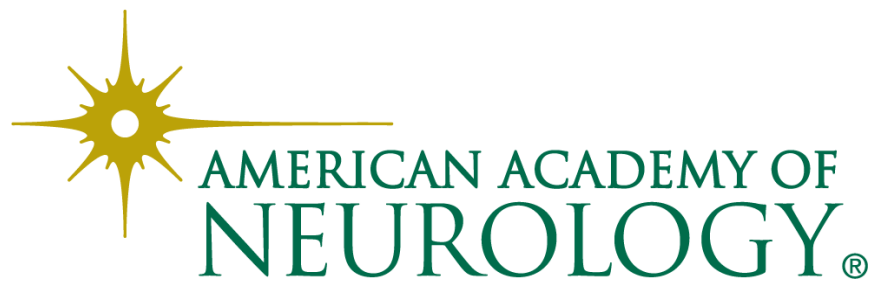

